Pacific Journal of Mathematics

SOME REMARKS ON THE COEFFICIENTS USED IN THE 


\title{
SOME REMARKS ON THE COEFFICIENTS USED IN THE THEORY OF HOMOLOGY MANIFOLDS
}

\author{
FRANK RAYMOND
}

\begin{abstract}
In the theory of generalized $n$-manifolds ( $n$-gms) or Cech cohomology manifolds ( $n$-cms), as developed principally by Wilder, the ring of coefficients had been a field. Due to the influence of transformation groups interest was aroused for more general coefficicnt systems. However, it is usually simpler to deal algebraically with coefficients in a field. Thus it becomes desirable to have a theorem which implies the validity of a result for $n$-cms over principal ideal domains when it is known to be valid for fields. The main result of our paper is devoted to such a theorem.
\end{abstract}

In [1; Chap. 1], and [5], it was shown that under suitable restrictions $X$ is an orientable $n$-cm over the integers $Z$ if $X$ is an orientable $n$-cm over $Z_{p}$, for all primes $p$ and $p=0$, (i.e. $Z_{0}$ is the field of quotients of $Z$ ). At the time it was not clear how to proceed to the general case of principal ideal domain $L$ instead of the integers $Z$. Theorem 1 and the Corollary, here, is the extension of this result to the general case and is, in fact, a strengthening of the earlier result even when $L=Z$. The proof is similiar to arguments of related results in [7]. The previous argument in [5] was given only in outline form and can be adopted, although not easily, to yield a proof of Theorem 1. However, on the basis of what was sketched there the argument would not be any shorter than that which we give here.

As a consequence of the method we establish (Theorem 2) the equivalence of singular homology $n$-manifolds and $n$-cms for a wider class of space than was given in [7] and [8].

Before proceeding to the preliminaries we would like to illustrate several of the advantages of Theorem 1. As was mentioned above, arguments involving fields as coefficients are considerably easier than those with a principal ideal domain as a coefficient system. A particular case in mind would be spectral sequence arguments. Thus under certain conditions the establishment of a result valid for $n$-cms over principal ideal domains where it is known to be valid for fields would be automatic by appealing to Theorem 1 and the Corollary. For example,

Received November 7, 1963, and in revised form July 13, 1964. Supported in part by National Science Foundation Grant GP-812 and U.S. Air Force Office of Scientific Research. 
Wilder's monotone mapping theorem is thereby extended. Also the proofs of Separation and Union theorems for $n$-gms with boundaries (Michigan Mathematical Journal 7, (1960) 7-21) would be considerably simplified and the factorization theorem (Theorem 6) becomes valid for arbitrary principal ideal domains instead of only the integers $Z$ and fields.

I wish to thank the referee for his helpful criticisms and suggestions on improving the exposition of a somewhat entagled earlier version of these results. I have incorporated his suggestions into the present version.

1. Preliminaries, All spaces will be Hausdorff. Two homology theories will eventually be used, the Borel-Moore homology theory with compact supports [2] and the singular homology theory. Coefficients will be taken in the $L$-module $G$, where $L$ is a principal ideal domain. A superscript " $s$ " will denote the singular homology if it becomes necessary to distinguish the two homology theories. The $p$ th Čech or sheaf cohomology module with compact supports and coefficients in $G$ of a locally compact space $X$ will be denoted by $H_{c}^{p}(X ; G)$. We shall assume familiarity with the concept of homology local connected up through dimension $n\left(l c_{n}\right.$ or $\left.l c_{n}^{s}\right)$ and cohomology locally connected (clc), (see [10] or [1]). For dimension of a locally compact space we shall use the Alexandroff-Cohen definition of cohomology dimension. Fundamental for the discussion will be the universal coefficient sequences.

$$
0 \longrightarrow H_{p}(X ; L) \otimes G \longrightarrow H_{p}(X ; G) \longrightarrow H_{p-1}(X ; L) * G \longrightarrow 0,
$$

and

$$
0 \longrightarrow H_{c}^{p}(X ; L) \otimes G \longrightarrow H_{c}^{p}(X ; G) \longrightarrow H_{c}^{p+1}(X ; L) * G \longrightarrow 0 \text {. }
$$

The sequences are exact and functorial.

(1.3) A singular homology $n$-manifold ( $n$-shm) Xover an L-module $G$ is a space such that

1. $\operatorname{dim}_{L} X<\infty$,

2. $H_{p}^{s}(X, X-x ; G)=\left\{\begin{array}{l}0, p \neq n \\ G, p \neq n\end{array}\right.$, for each $x \in X$,

3. There exists a covering by open sets $\left\{U_{\alpha}\right\}$ of $X$ such that

$$
j_{*}: H_{n}^{s}\left(X, X-\bar{U}_{\alpha} ; G\right) \longrightarrow H_{n}^{s}(X, X-x ; G)
$$

is bijective for each $x \in U_{\alpha}$.

This is what I called locally orientable singular homology $n$-mani- 
fold over $G$ in [7]. It isn't hard to see that 2 and 3 imply that $X$ is locally a Peano-space and consequently condition 1 makes sense, (that is, $X$ is therefore locally compact and dimension is consequently well defined). We say that $X$ is orientable if for each open connected set $O$, with compact closure, the homomorphism $j_{*}: H_{n}^{s}(X, X-\bar{O} ; G) \rightarrow$ $H_{n}^{r}(X, X-x ; G)$ is bijective, for all $x \in O$. That is, 2 and 3 say that the orientation sheaf or the sheaf of local homology groups is trivial in all dimensions except $n$ and there it is locally constant. It is orientable, therefore, if the orientation sheaf is constant.

By a generalized $n$-manifold over $L(n$-gm) we shall mean a $\check{C} e c h$ cohomology $n$-maniford over $L(n-\mathrm{cm})$. This is a locally orientable generalized $n$-manifold in the sense of Wilder and with coefficients over $L$. See for example, [10], [1], [2], and [5].

(1.4) In [2], Borel and Moore defined a homology theory for locally compact spaces. This can be regarded as a single space theory in terms of Eilenberg and Steenrod [3; Chap. 10]. However, by looking at the homology theory as a relative homology theory (see below) we shall easily reformulate the definition of (Borel-Moore) homology manifolds to one that is analogous to that already given for singular homology manifolds. This enables us to use a single argument for the proof of Theorem 1 valid for both homology theories. Furthermore, the comparison between singular homology manifolds and homology manifolds there, in a sense, reduces to a comparison of the respective homology theories (Theorem 2). (An alternative would be to reformulate the definition of singular homology manifold in terms of locally finite singular homology leaving intact the Borel-Mioore definition. But for various reasons we do not think this procedure is preferable.)

Let $U$ be an open subset of the locally compact space $X$. Define the relative group

$$
H_{p}(X, X-U ; G) \text { to be } H_{p}(U ; G),
$$

where $H_{p}(U ; G)$ denotes the Borel-Moore homology theory with closed supports, [cf. 2; 3.8 and $\S 5]$. Thus, from the single space theory of Borel-Moore homology we obtain a homology theory, in the sense of Eilenberg and Steenrod, defined on the category of closed locally compact pairs and proper maps. We now follow Eilenberg and Steenrod and extend the relative theory on compact pairs to arbitrary Hausdorff pairs.

Let $(X, Y)$ be an arbitrary Hausdorff pair. Define

$$
H_{p}^{c}(X, Y ; G) \text { to be } \operatorname{Dir} \lim _{\lambda} H_{p}\left(X_{\lambda}, A_{\lambda} ; G\right)
$$

where $\left(X_{\lambda}, A_{\lambda}\right)$ runs through all compact pairs (direction is given by inclusion) in the arbitrary pair $(X, Y)$. This procedure extends the 
homology theory from compact pairs to arbitrary pairs. See [3; p. 255] for outline of details. In particular, if $X$ is locally compact and $Y$ a subset whose closure is compact then

(i ) $\operatorname{Dir} \lim _{U \supset Y} H_{p}(U)=\operatorname{Dir} \lim _{U \supset Y} H_{p}(X, X-U)=H_{p}^{c}(X, X-Y)$, where $U$ runs through the open neighborhoods of the subspace $Y$. Clearly,

(ii) $H_{p}(X, X-U)=H_{p}^{c}(X, X-U)$, when $X$ is locally compact, $U$ is open and $\bar{U}$ is compact, (use excision). We shall call this homology theory, $H_{*}^{c}$, defined on arbitrary Hausdorff pairs and continuous maps the Borel-Moore homology theory with compact carriers. For the pair $(X, Y)$ we have, of course, the exact sequence

$$
\begin{aligned}
& \longrightarrow H_{p}^{c}(Y ; G) \stackrel{i_{*}}{\longrightarrow} H_{p}^{c}(X ; G) \stackrel{j_{*}}{\longrightarrow} H_{p}^{c}(X, Y ; G) \\
& \stackrel{\partial^{*}}{\longrightarrow} H_{p-1}^{c}(Y ; G) \longrightarrow \text {. }
\end{aligned}
$$

For locally compact spaces homology $n$-manifolds $(n-\mathrm{hm})$ over an $L$-module $G$ are defined exactly as singular homology $n$-manifolds over $G$ were defined in 1.3. The homology to be used is the Borel-Moore homology theory with compact carriers. Putting $Y=$ point in (i) above we easily see that the definition is equivalent to that in $[2 ; 7.5]$ when $X$ is $l c_{0}$.

Poincaré duality holds for homology manifolds. The form of duality we shall need is that with compact supports. Thus it is necessary to identify the Borel-Moore homology with compact carriers with the homology of the Borel-Moore chains with compact support. If $U$ is an open subset of the locally compact space $X$ we have the exact sequence of Borel-Moore chains with compact support

$$
\begin{aligned}
0 \longrightarrow & \Gamma_{c}\left(\mathscr{C}_{H}(X ; L) \mid U \otimes G\right) \longrightarrow \Gamma_{b}\left(\mathscr{C}_{H}(X ; L) \otimes G\right) \\
& \longrightarrow \Gamma_{c}\left(\mathscr{C}_{H}(X ; L) \otimes G\right) / \Gamma_{c}\left(\mathscr{C}_{H}(X ; L) \mid U \otimes G\right) \longrightarrow 0,
\end{aligned}
$$

where $\mathscr{C}_{H}(X ; L)$ denotes the standard sheaf for homology on $X$ of $[2 ; \S 3]$. The derived homology sequence is analogous to (iii) above, with $Y$ replaced by $U$, compare $[9 ; 2.2]$. However, more is true. The chains with compact support in $U, \Gamma_{c}\left(\mathscr{C}_{H}(X ; L) \mid U \otimes G\right)$, can be identified with the direct limit, directed by inclusion, of all the chains of $X$ whose support lie in compact subsets of $U$. On the other hand, the chains of $X$ whose support lie in a compact subset, $F$, have homology functorially equivalent to the homology of $F$. See [2; 3.4] and [9] for details. Similarly we may identify $H_{*}^{c}(X, U ; G)$ as the homology of the chains of $X$ with compact support mod those chains of $X$ whose support, again compact, lies in $U$.

In what follows we shall only use the Borel-Moore homology theory for locally compact pairs $(X, U), U$ open in $X$, and with compact 
carriers (or the equivalent, with compact supports). Therefore we shall simply abbreviate $H_{*}^{c}(X ; U)$ to $H_{*}(X, U)$.

(1.5) Let $q$ stand ambiguously for a prime ideal, $\neq 0$, or any of its generators of a principal ideal domain $L$. The ideal is maximal and the residue class ring is a field and will be denoted by $L_{q}$. Denote the field of quotients of $L$ by $L_{0}$. Define the family of all fields determined by $L$ to be the collection of fields just described. Denote the collection by $\mathscr{S}$.

(1.6) The following statements are well known consequences of elementary properties of tensor and Tor functors applied to L-modules.

Suppose that $G$ is an L-module and that $G \otimes L_{0}=0$, then $G$ contains only torsion elements.

Suppose that $G$ is L-module, and $q$ is a prime in L. Then $G$ contains q-torsion, if and only if, $G * L_{q} \neq 0$. Hence, if $G$ and $H$ contain $q$-torsion, $G * H \neq 0$, because $G$ contains a cyclic submodule isomorphic to $L_{q}$ and $L_{q} * H \rightarrow G * H$ is injective.

Let $g \in G$, and $(g)$ be the cyclic submodule generated by $g$. From the exact sequence

$$
G /(g) * G \longrightarrow(g) \otimes G \longrightarrow G \otimes G,
$$

and the fact that $G /(g) * G$ is a torsion module, $(g)$ can not be isomorphic to $L$ if $G=0$. Hence, if $G \otimes G=0$, then $G$ contains only torsion elements. In particular, if $G \otimes G=0$ and $G \neq 0$, then $G * G \neq 0$.

(1.7) Let $X$ be locally compact, $U$ and $V$ open subsets of $X$ such that $\bar{V} \subset U$, and $\bar{V}$ is compact. Suppose that $X$ is homology locally connected up through dimension $n$ in terms of the Borel-Moore homology theory with compact supports and coefficients in $L, l c_{n}$ (respectively; $X$ is $l c_{n}^{s}$ over $L$ ). Then the images of the homomorphisms

$$
\begin{aligned}
& i_{*}: H_{p}(V ; L) \longrightarrow H_{p}(U ; L) \\
& j_{*}: H_{p}(X, X-\bar{V} ; L) \longrightarrow H_{p}(X, X-\bar{U} ; L)
\end{aligned}
$$

are finitely generated, for all $p \leqq n$ in terms of the Borel-Moore homology theory with compact supports (respectively; in terms of the singular homology).

Proof. This argument has been communicated to the author by C. N. Lee. Both the Borel-Moore homology with compact supports and 
the singular homology theory satisfy the Mayer-Vietoris sequence in terms of open subsets. The absolute case for singular homology then becomes an exact copy of the absolute case for the Borel-Moore homology theory which is proved in $[2 ; 6.8]$. The relative case now follows from exactness and a little diagram chasing.

2. The family $\mathscr{L}^{*}$. Each field $L_{p} \in \mathscr{L}(p=0$, or $q)$ is a vector space over the rationals or the integers modulo a prime. This is determined, of course, by the characteristic of each $L_{p}$. Denote the corresponding collection of fields isomorphic to the integers modulo a prime and possibly the rationals by $\mathscr{L}^{*}$. Clearly, $L_{p}$ is a $L_{p}^{*}$ module.

Let $\chi(L)$ denote the characteristic of $L$. In general we have three cases:

1. $\chi(L)=m, m \neq 0$.

2. $\chi(L)=0, \chi\left(L_{p}\right)=0$, for all $L_{p} \in \mathscr{L}$.

3. $\chi(L)=0, \chi\left(L_{p}\right) \neq 0$, for some $L_{p} \in \mathscr{L}$.

The structure of $\mathscr{L}^{*}$ for these three cases are respectively:

1. $Z_{m}=\mathscr{L}^{*}$, and $L$ is additively a vector space over $Z_{m}$.

2. $Z_{0}=\mathscr{L}^{*}$, and $L$ is additively a vector space over $Z_{0}$.

3. $Z_{0}$ and all $Z_{m}$ for which there exists an $L_{p} \in \mathscr{L}$ for which $\chi\left(L_{p}\right)=m \neq 0$.

It is the presence of the third case which causes the difficulties in the main theorems.

The first observation to be made is that $\operatorname{dim}_{G} X \leqq \operatorname{dim}_{L} X$, for any $L$-module $G$. Also, if $G$ is free over $L$, then $\operatorname{dim}_{G} X=\operatorname{dim}_{L} X$. This is a direct consequence of 1.2. Hence, $\operatorname{dim}_{L_{p}} X=\operatorname{dim}_{L_{p}^{*}} X$, and, in fact for Cases 1 and $2, \operatorname{dim}_{L} X=\operatorname{dim}_{L_{p}^{*}} X$, for all $L_{p}^{*} \in \mathscr{L}^{*}$.

The next proposition is also an easy consequence of the universal coefficient Theorems 1.1 and 1.2.

(2.1) Proposition. A space $X$ is a $n$-cm, $n$-shm or an $n$-hm over $L$ (respectively; orientable) where $L$ is a field of characteristic $m$, if and only if, $X$ is an $n$-cm, $n$-shm or an $n$-hm over $Z_{m}$, (respectively; orientable).

3. A dimension lemma. This section is concerned with the determination of $\operatorname{dim}_{L} X$ when one knows $\operatorname{dim}_{L_{p}} X$ or $\operatorname{dim}_{L_{p}^{*}} X$ for all $L_{p} \in \mathscr{L}$, or all $L_{p}^{*} \in \mathscr{L}^{*}$. Define $\operatorname{dim}_{\mathscr{C} *} X=\max _{L_{p}^{*} \in \mathscr{L}^{*}}\left\{\operatorname{dim}_{L_{p}^{*}} X\right\}$. This will be called the field dimension of $X$. Clearly, $\operatorname{dim}_{\mathscr{P} *} X=\operatorname{dim}_{\mathscr{E}} X$, where $\operatorname{dim}_{\mathscr{L}} X$ is defined analogously.

(3.1) Lemma. Let $X$ be locally compact. Then $\operatorname{dim}_{L} X \leqq$ $\left(\operatorname{dim}_{\mathscr{S}^{*}} X\right)+1$. Furthermore, the strict inequality holds for Cases 1 and 2 of $\S 2$. 
Proof. By the remarks in $\S 2, \operatorname{dim}_{L} X=\operatorname{dim}_{L_{p}^{*}} X=\operatorname{dim}_{L_{p}} X=$ $\operatorname{dim}_{\mathscr{L}^{*}} X$ for Cases 1 and 2 .

Suppose now that $n=\operatorname{dim}_{\mathscr{C} *} X<\operatorname{dim}_{L} X$. (We are necessarily in Case 3). Then $H_{c}^{n+k}\left(U ; L_{p}\right)=0$, for all $L_{p} \in \mathscr{S}$, all open $U \subset X$, and all $k>0$. By $1.2, H_{c}^{n+k}(U ; L) \otimes L_{p}=0$ and $H_{c}^{n+k+1}(U ; L) * L_{p}=0$, for all $L_{p} \in \mathscr{L}$. Hence by $1.6, H_{c}^{n+k+1}(U ; L)=0$, for all $k>0$, and all open subsets $U, U \subset X$. But if $H_{c}^{n+1}(U ; L)=0$, for all $U$, then $\operatorname{dim}_{L} X \leqq n$. Therefore, there exists some open subset $U$ such that $H_{c}^{n+1}(U ; L) \neq 0$. This implies that $H_{c}^{n+1}(U ; L)$ is a torsion module. (In fact, $H_{c}^{n+1}(V ; L)$ is a torsion module, possibly trivial, for all open subsets $V$ of $X$.) We observe that if $\operatorname{dim}_{L} X>\operatorname{dim}_{\mathscr{C}^{*}} X=n, \operatorname{dim}_{L} X=$ $n+1$. Hence, there exists an $L_{q} \in \mathscr{L}$ such that $\operatorname{dim}_{\mathscr{S} *} X=\operatorname{dim}_{L_{q}} X$ and there exists an open subset $V$ such that $H_{c}^{n+1}(V ; L)$ has nontrivial $q$-torsion. Note also that if $\operatorname{dim}_{L_{p}} X<\operatorname{dim}_{L_{0}} X$ for all $L_{p} \in \mathscr{L}, L_{p} \neq L_{0}$, then $\operatorname{dim}_{L} X=\operatorname{dim}_{L_{0}} X=\operatorname{dim}_{\mathscr{L}^{*}} X$.

Actually the lemma is stronger than we need. We shall only use in Theorem 1 the fact that $\operatorname{dim}_{L} X<\infty$ if $\operatorname{dim}_{\infty} X<\infty$. However, it can be used to simplify and/or extend several arguments in dimension theory. The following is a sample of such a situation.

Corollary. (Bockstein, and Fary [4].) Let $X$ be a locally compact Hausdorff space such that $\operatorname{dim}_{L} X=n<\infty$. Then $\operatorname{dim}_{L} X^{k}=k n$ or $k(n-1)+1$. The latter case holds, if and only if, $\operatorname{dim}_{\mathscr{S}^{*}} X<\operatorname{dim}_{L} X$.

Proof. If $\operatorname{dim}_{L} X=\operatorname{dim}_{\mathscr{S}^{*} *} X$, then $\operatorname{dim}_{L} X^{k}=k n=\operatorname{dim}_{\mathscr{C}^{*}} X^{k}$. (It is known that $\operatorname{dim}_{L} X \times Y \leqq \operatorname{dim}_{L} X+\operatorname{dim}_{L} Y$, see [1, p. 15]. There are other published proofs of this fact that seem to be incomplete; however, they can be completed if one assumes $\operatorname{dim}_{L} X \times Y<\infty$. Now, $\operatorname{dim}_{\mathscr{L}_{*}} X^{k} \geqq k \operatorname{dim}_{\mathscr{S} *} X$ follows from repeated use of the Künneth theorem and $\operatorname{dim}_{L} X^{k}=k \operatorname{dim}_{L} X$ from the fact that $\operatorname{dim}_{L} X^{k} \geqq \operatorname{dim}_{\mathscr{C}^{*}} X^{k}$ ). If $\operatorname{dim}_{L} X^{k} \neq k n$, then $\operatorname{dim}_{L} X>\operatorname{dim}_{\mathscr{P} *} X$. Then there exists some "prime" $q \in L$ such that $\operatorname{dim}_{L_{q}^{*}} X=\operatorname{dim}_{\mathscr{S}^{*}} X=n-1$.

In particular we can find an open subset $U \subset X$ such that $H_{c}^{n}(U ; L)$ contains $q$-torsion. Thus, for $k=1$, the theorem is proved. We proceed by induction. Assume $H_{c}^{r(n-1)+1}\left(U^{r} ; L\right)$ has $q$-torsion, for all $r<k$. Then

$$
H_{c}^{r(n-1)+1}\left(U^{r} ; L\right) * H_{c}^{n}(U ; L) \neq 0, \quad \text { by } 1.6 .
$$

Hence, by the Künneth theorem $H_{c}^{r(n-1)+1+n-1}\left(U^{r} \times U ; L\right) \neq 0$. Setting $r=k-1$, we obtain $H_{c}^{k(n-1)+1}\left(U^{k} ; L\right) \neq 0$. Since $\operatorname{dim}_{\mathscr{S}^{*}} X^{k}=k(n-1)$, $\operatorname{dim}_{L} X^{k}=k(n-1)+1$.

It is easily shown that the latter case cannot hold if $X$ is clc over $L$, the point being $\operatorname{dim}_{L} X=\operatorname{dim}_{\mathscr{L} *}$ when $X$ is clc. 
4. The main results.

Theorem 1. Let $X$ be $l c_{n}$ (respectively; $\left.l c_{n}^{s}\right)$ over L. Then $X$ is an $n$-hm (respectively; an $n$-shm) over $L$, if and only if, $X$ is an $n$-hm (respectively; an $n$-shm) over each $L_{p}^{*} \in \mathscr{L}^{*}$. Moreover, $X$ is orientable, if and only if, $X$ is orientable over each $L_{p}^{*} \in \mathscr{L}^{*}$.

TheOREM 2. Let $X$ be $l c_{n}$ (respectively; $l c_{\infty}^{s}$ ) over $L$, then $X$ is an $n-\mathrm{cm}$, if and only if $X$ is an $n-\mathrm{hm}$ (respectively; $n$-shm) over $L$. Moreover $X$ is an orientatable $n$-cm if and only if $X$ is an orientable $n$-hm (respectively; $n$-shm).

CoRollary. Let $X$ be $l c_{n}$ over $L$ then $X$ is an $n$-cm over $L$ if and only if $X$ is an $n$-cm over every $L_{p}^{*} \in \mathscr{L}^{*}$.

Summarizing these results we have:

Under $l c_{n}$ over $L, n-\mathrm{cm}_{L} \Leftrightarrow n-\mathrm{cm}_{\mathscr{L} *} \Leftrightarrow n-\mathrm{cm}_{\mathscr{L}} \Leftrightarrow n-\mathrm{hm}_{L}$

$$
\Leftrightarrow n-\mathrm{hm}_{\mathscr{L}} \Leftrightarrow n-\mathrm{hm}_{\mathscr{L} *} \text {. }
$$

Under lc $c_{n}^{s}$ over $L, n$-shm $\mathrm{s}_{L} \Leftrightarrow n$-shm $\mathrm{s}_{\mathscr{L} *}\left\langle n\right.$-shm $\mathrm{s}_{\mathscr{L}}$.

Under lc over $L, n-\mathrm{cm}_{L} \Leftrightarrow n-\mathrm{cm}_{\mathscr{L} *} \Leftrightarrow n-\mathrm{cm}_{\mathscr{L}} \Leftrightarrow n-\mathrm{hm}_{L}$

$\Leftrightarrow n-\mathrm{hm}_{\mathscr{L}}<\Rightarrow n-\mathrm{hm}_{\mathscr{L}} * \Rightarrow n-\mathrm{shm}_{L}$

$\Leftrightarrow n-\operatorname{shm}_{\mathscr{L}}\left\langle\Rightarrow n-\operatorname{shm}_{\mathscr{L}}\right.$.

Proof of Theorem 1. If $X$ is an $n$-hm (respectively; an $n$-shm) over $L$, the universal coefficient theorems immediately imply that $X$ is an $n$-hm (respectively; an $n$-shm) over each $L_{n} \in \mathscr{S}$, and over each $L_{p}^{*} \in \mathscr{L}^{*}$.

For the converse we shall not distinguish the two respective cases as the arguments are identical. The homology groups are to be interpreted as the singular homology or the Borel-Moore homology with compact supports as the case may be.

Assume that $X$ is an $n$-hm (or an $n$-shm) over each $L_{p}^{*} \in \mathscr{L}^{*}$. Then as $L_{p}$ is a vector space over $L_{p}^{*}$, we can (universal coefficient theorems) assume that $X$ is an $n$-hm (or an $n$-shm) over each $L_{p} \in \mathscr{L}$.

Next, we show that $\operatorname{dim}_{L_{p}} X=n$, for all $p$. Hence by $\S 3$, $\operatorname{dim}_{L} X \leqq n+1$. In fact, after we show that $X$ is an $n$-hm (or $n$-shm) over $L$, we may conclude that $\operatorname{dim}_{L} X=n$. These statements follow from the lemma:

Let $X$ be $l c_{0}$ over the principal ideal domain $L$ or have a countable base for its topology (respectively: no assumptions if the singular homology is used). If $X$ is an $n-\mathrm{hm}$ (respectively: $n$-shm) then $\operatorname{dim}_{L} X=n$. 
The proof of this general proposition depends upon the fact that for finite dimensional spaces over $L$, $\operatorname{dim}_{L}$ is a local property. That is, one need only check the vanishing of the cohomology modules for sufficiently small subsets of $X$. The hypothesis guarantees that the $(-1)^{\text {st }}$-homology modules for the Borel-Moore homology theory are 0 for open subsets of $X$. We now use Poincaré duality, (Poincaré duality is proved for singular homology manifolds in [7]), and obtain that $H_{c}^{n+k}(U ; L)=H_{-k}(U ; L)=0$, for all open subsets $U$ within an orientable part of $X$ and all $k>0$. This completes the proof of the lemma.

Let $x \in X$, then $H_{k}\left(X, X-x ; L_{p}\right)=0$, for all $k \neq n$. In particular, $H_{k}(X, X-x ; L) \otimes L_{0}=H_{k-1}(X, X-x ; L) * L_{q}=0$, for all $k \neq n$, and all $q$. Thus, for $1.6, H_{k}(X, X-x ; L)=0$, for all $k \neq n$ or $n-1$. Furthermore, for any open subset $P \subset X, H_{n}(X, P ; L)$ is torsion free since $H_{n+1}\left(X, P ; L_{q}\right)=0$, by Poincaré duality. Let $U$ be a connected neighborhood of $x$ (with compact closure) such that $U$ is orientable with respect to $L_{0}$. Let $V$ be any connected open subset of $U$ such that $\bar{V} \subset U$; let $F$ denote the image of

$$
j_{*}: H_{n}(X, X-\bar{U} ; L) \longrightarrow H_{n}(X, X-\bar{V} ; L) .
$$

The module $F$ is a finitely generated (by 1.7 ) torsion free $L$-module. Since

$$
\begin{gathered}
j_{*}: H_{n}(X, X-\bar{U} ; L) \otimes L_{0} \longrightarrow H_{n}(X, X-\bar{V} ; L) \otimes L_{0} \\
=H_{n}\left(X, X-\bar{V} ; L_{0}\right)
\end{gathered}
$$

is bijective and has image isomorphic to $L_{0}$, it follows that $F$ must be precisely isomorphic to $L$. Now tensor (4.1) by $L_{q}$ and obtain the commutative diagram

$$
\begin{aligned}
& H_{n}(X, X-\bar{U} ; L) \otimes L_{q} \longrightarrow F \otimes L_{q} \longrightarrow 0 \\
& \bigcup_{\left(j_{*} \otimes 1\right)} \downarrow \\
& H_{n}(X, X-\bar{V} ; L) \otimes L_{q}
\end{aligned}
$$

which is exact on the top row. Since $F \otimes L_{q} \cong L_{q}$,

$$
H_{n}(X, X-\bar{U} ; L) \otimes L_{q} \neq 0,
$$

and consequently $H_{n}\left(X, X-\bar{U} ; L_{q}\right) \cong L_{q}$. Therefore, $U$ is an orientable $n$-hm or $n$-shm over $L_{q}$, for all $q$. Note further that

$$
H_{n-1}(X, X-\bar{U} ; L) * L_{q}=0 \text {. }
$$

Therefore it follows that $H_{n-1}(X, X-x ; L)=0$, since it is the direct limit of torsion free modules (such as $H_{n-1}(X, X-\bar{U} ; L)$ ) and $H_{n-1}(X, X-x ; L) \otimes L_{0}=0$.

We show now that $j_{*}: H_{n}(X, X-\bar{U} ; L) \rightarrow H_{n}(X, X-\bar{V} ; L)$ is 
bijective with image, $F$, isomorphic to $L$, whence the orientation sheaf is locally isomorphic to $L$ in dimension $n$, (i.e. $j_{*}: H_{n}$ ) $\left.X, X-\bar{U} ; L\right) \rightarrow$ $H_{n}(X, X-x ; L)$ is bijective for all $\left.x \in U\right)$.

First, $H_{n}(X-\bar{V}, X-\bar{U} ; L)$ is torsion free, and $H_{n}\left(X-\bar{V}, X-\bar{U} ; L_{p}\right) \cong$ $H_{c}^{0}\left(\bar{U}-\bar{V} ; L_{p}\right)=0$. Therefore by 1.1 and $1.6 H_{n}(X-\bar{V}, X-\bar{U} ; L)=0$. Exactness of the triple $(X, X-\bar{V}, X-\bar{U})$ implies that $j_{*}$ is injective. A similar argument shows that $H_{n}(X, X-\bar{V} ; L)$ is also isomorphic to $L$. Now, $H_{n}\left(X-\bar{V}, X-\bar{U} ; L_{q}\right)=0$, for all $L_{q} \in \mathscr{L}$, implies $H_{n-1}(X-\bar{V}, X-\bar{U} ; L)$ is torsion free. Thus $j_{*}$ must also be surjective.

We have shown that $X$ is an $n$-hm (or $n$-shm) over $L$. That $X$ is orientable over $L$ if and only if it is orientable over each $L_{p} \in \mathscr{L}$ is clear from the above. In fact, orientability over $L_{0}$ is equivalent to orientability over $L$.

It is interesting to note that $l c_{0}$ was used to make the $(-1)^{s t}$-BorelMoore homology groups vanish and to guarantee sufficiently many open connected subsets of $X$. (These facts are implied by Condition 3 of 1.3 in the singular case). The $l c_{n}$ (and $l c_{n}^{s}$ ) condition over $L$ was used to imply the finite generation of the image of $j_{*}$ in 4.1 (Added in proof: $l c_{n}$ also guarantee the validity of change in rings.) $^{2}$

Proof of Theorem 2. Suppose $X$ is an $n$-cm over $L$ then Poincare duality implies $H_{k}(X, X-x ; L) \cong H^{n-k}(x ; L)=0$, for all integers $k \neq n$. In dimension, $n, H_{n}(X, X-\bar{V} ; L) \cong H_{c}^{0}(\bar{V} ; L) \cong L$, if $V$ is connected contained within an orientable part of $X$ and $\bar{V}$ compact. Furthermore, duality is functorial with respect to inclusion. Thus $X$ is an $n$-hm and orientable if and only if $X$ is orientable as an $n-\mathrm{cm}$. [cf $2 ; 7.12$ for a proof that essentially uses only the universal coefficient theorem]. Note also that if $X$ is an $n-\mathrm{cm}$ over $L$, then $X$ is an $n-\mathrm{cm}$ over $L_{p}$ and $L_{p}^{*}$, for all $L_{p} \in \mathscr{L}$, and $L_{p}^{*} \in \mathscr{S}^{*}$. This follows trivially from the universal coefficient sequences. In particular, $X$ is an $n$-hm over all $L_{p} \in \mathscr{L}^{*}$. In [8], I have shown that if $X$ is $l c_{\infty}^{s}$ over $L_{p}^{*}$, and is an $n$-hm over $L_{p}^{*}$, then $X$ is an $n$-shm over $L_{p}^{*}$. (The point being that the relative Borel-Moore homology groups $H_{*}\left(X, U ; L_{p}^{*}\right)$ are naturally equivalent to the Čech homology groups (with compact carriers) which are naturally equivalent to the relative singular homology groups $H_{*}^{s}\left(X, U ; L_{p}\right)$. Therefore, if $X$ is a cm over $L$ and is $l c_{\infty}^{s}$ over $L$ (hence $l c_{\infty}^{s}$ over every $L_{p}^{*}$ ), then, by Theorem $1, X$ is an $n$-shm over $L$.

On the other hand, if $X$ is an $n-\mathrm{hm}$ and is $l c_{n}$ (or an $n$-shm and is $\left.l c_{n}^{s}\right)$ over $L$, then Poincaré duality (image $H_{p}(V ; L) \rightarrow H_{p}(U ; L)$ is isomorphic to image $\left.H_{c}^{n-p}(V ; L) \rightarrow H^{n-p}(U ; L)\right)$ implies that $X$ is an $n$ $\mathrm{cm}$ over $L$. (The argument is that of $[2 ; 7.12]$ with the observation that $l c_{n}$ condition suffices instead of the full $l c_{\infty}$ because $H_{c}^{n-p}$ is 0 for negative $p$.) 
Again orientability statements are clear.

Proof of the corollary. Trivially, if $X$ is an $n$-cm over $L$ then $X$ is an $n$-cm over every coefficient $L$-module. Conversely, if $X$ is an $n$-cm over $L_{p}^{*}$ then $X$ is an $n$-hm over $L_{p}^{*}$, by Theorem 2. By Theorem 1 , if $X$ is, in addition, $l c_{n}$ over $L$, then $X$ is an $n$-hm over $L$. Again by Theorem $2, X$ is an $n$-cm over $L$.

In [8] it was observed that Theorem 2 held for fields. In [6], the "if" part (singular homology) of Theorem 2 for $L=Z$ and $X$ having a countable basis is proved. Their argument, however, does not appear to be amenable to the general case because of the reliance upon duality between compact topological groups and discrete abelian groups.

In Theorem 1 , the integer $n$ was kept fixed, i.e. $n$ was independent of $L_{p}, L_{p} \in \mathscr{L}$. In order to free the theorem from this assumption a strengthening of the other hypotheses is needed, although no examples implying the contrary are known to me. An illustration of (possibly) stronger hypotheses which would imply the constancy of the integer $n$ would be to assume that $H_{n_{0}}(X, X-x ; L)$ is finitely generated for some $x \in X$, where $n_{0}=\operatorname{dim}_{L_{0}} X$, or to assume that $\operatorname{dim}_{L_{0}} X=\operatorname{dim}_{\mathscr{L}^{*}} X$. (Cf. [1; I, 4.11]).

One could formulate these remarks as a question. Let $X$ be clc (respectively: $l c_{\infty}^{s}$ ) over $L$, and $\operatorname{dim}_{L} X<\infty$. Suppose $X$ is $n_{p}$-cm (respectively: an $n_{p}$-shm) over each $L_{p} \in \mathscr{L}$. Then, is $X$ an $n$-cm (respectively: an $n$-shm) over $L$, for some integer $n$ ? It seems likely that the answer is always affirmative. (We have already seen that if the characteristic of $L$ satisfies 1 or 2 of $\S 2$, the answer is affirmative.) However, if the local connectedness assumptions over $L$ were removed it seems likely that the answer would be negative, (if not then it would follow that every compact group acting effectively on a manifold would necessarily be a Lie group).

Added in proof. If $L^{\prime}$ is a module over $L$ and both are principal ideal domains then $H_{\rho}\left(X, X-\bar{U} ; L^{\prime}\right)$ has the interpretations depending upon which ring, $L$ or $L^{\prime}$, is used for the ground ring. The interpretations agree, that is the change of rings is valid, for the singular homology as is well known, and for the Borel-Moore homology theory, with compact carriers, if $X$ is $l c^{p}$ over $L$, (due to Bredon). We have implicitly used the validity of the change of rings in the proof of Theorem 1. When $L^{\prime}$ is a field, which is all that we need, the validity for the Borel-Moore homology can easily established as follows. Let the $L^{\prime}$-modules

$$
\operatorname{Hom}_{L^{\prime}}\left(\Gamma_{o}\left(\mathscr{C}_{H}(X ; L) \mid U \otimes_{K} L^{\prime}\right), L^{\prime}\right) \text { and } \operatorname{Hom}_{L^{\prime}}\left(\Gamma_{o}\left(\mathscr{C}_{H}\left(X ; L^{\prime}\right) \mid U\right), L^{\prime}\right)
$$

determine presheafs. Apply the arguments of [2;6.4 and 6.6] to both presheafs. This implies that they both determine the sheaf-theoretic 
cohomology of $U$, with closed supports, over $L$ as dual spaces of both homology theories. Hence the change of rings is valid. A recent example of Bredon, which has also inspired these remarks, shows that this change of rings is not valid without the $l c^{p}$ hypothesis. A complete discussion of the change in rings will appear in a forthcoming book of Bredon on sheaf theory.

\section{REFERENCES}

1. A. Borel et al., Seminar in transformation groups, annals study 46, Princeton, 1960.

2. A. Borel and J. C. Moore, Homology theory for locally compact spaces, Mich. Math. Jour. 7 (1960), 137-160.

3. S. Eilenberg and N. Steenrod, Foundations of Algebraic Topology, Princeton University Press, 1952.

4. I. Fary, Dimension of the square of a space, Bull. Amer. Math. Soc. 67 (1961), 135-137.

5. Kyung Whan Kwun and Frank Raymond, Generalized cells in generalized manifolds, Proc. Amer. Math. Soc. 11 (1960), 135-139.

6. D. Montgomery and D. Mostow, Toriod transformation groups on euclidean spaces, Ill. Journ. of Math. 2 (1958), 459-481.

7. Frank Raymond, Poincaré duality for homology manifolds, Doctoral dissertation, University of Michigan (1958), multicopy.

8. — A note on the local " $C$ " groups of Griffiths, Mich. Math. J. 7 (1960), $1-5$.

9. L Local cohomology groups with closed supports, Math. Zeit. 76 (1961), 31-41.

10. R. L. Wilder, Topology of manifolds, Amer. Math. Soc. Coll. Publ. 32, 1949.

UNIVERSITY OF CALIFORNIA, BERKELEY

AND

UNIVERSITY OF MICHIGAN 


\title{
PACIFIC JOURNAL OF MATHEMATICS
}

\author{
EDITORS
}

\author{
H. SAmelson \\ Stanford University \\ Stanford, California \\ R. M. Blumenthal \\ University of Washington \\ Seattle, Washington 98105
}

\author{
J. DugundjI \\ University of Southern California \\ Los Angeles, California 90007
}

*Richard Arens

University of California

Los Angeles, California 90024

\section{ASSOCIATE EDITORS}
E. F. BECKENBACH
B. H. NeUmanN
F. WOLF
K. YoSIDA

\section{SUPPORTING INSTITUTIONS}

\author{
UNIVERSITY OF BRITISH COLUMBIA \\ CALIFORNIA INSTITUTE OF TECHNOLOGY \\ UNIVERSITY OF CALIFORNIA \\ MONTANA STATE UNIVERSITY \\ UNIVERSITY OF NEVADA \\ NEW MEXICO STATE UNIVERSITY \\ OREGON STATE UNIVERSITY \\ UNIVERSITY OF OREGON \\ OSAKA UNIVERSITY \\ UNIVERSITY OF SOUTHERN CALIFORNIA
}

\author{
STANFORD UNIVERSITY \\ UNIVERSITY OF TOKYO \\ UNIVERSITY OF UTAH \\ WASHINGTON STATE UNIVERSITY \\ UNIVERSITY OF WASHINGTON \\ * * * * \\ AMERICAN MATHEMATICAL SOCIETY \\ CALIFORNIA RESEARCH CORPORATION \\ SPACE TECHNOLOGY LABORATORIES \\ NAVAL ORDNANCE TEST STATION
}

Mathematical papers intended for publication in the Pacific Journal of Mathematics should by typewritten (double spaced). The first paragraph or two must be capable of being used separately as a synopsis of the entire paper. It should not contain references to the bibliography. No separate author's resumé is required. Manuscripts may be sent to any one of the four editors. All other communications to the editors should be addressed to the managing editor, Richard Arens, at the University of California, Los Angeles, California 90024.

50 reprints per author of each article are furnished free of charge; additional copies may be obtained at cost in multiples of 50 .

The Pacific Journal of Mathematics is published quarterly, in March, June, September, and December. Effective with Volume 13 the price per volume (4 numbers) is $\$ 18.00$; single issues, $\$ 5.00$. Special price for current issues to individual faculty members of supporting institutions and to individual members of the American Mathematical Society: $\$ 8.00$ per volume; single issues $\$ 2.50$. Back numbers are available.

Subscriptions, orders for back numbers, and changes of address should be sent to Pacific Journal of Mathematics, 103 Highland Boulevard, Berkeley 8, California.

Printed at Kokusai Bunken Insatsusha (International Academic Printing Co., Ltd.), No. 6, 2-chome, Fujimi-cho, Chiyoda-ku, Tokyo, Japan.

PUBLISHED BY PACIFIC JOURNAL OF MATHEMATICS, A NON-PROFIT CORPORATION

The Supporting Institutions listed above contribute to the cost of publication of this Journal, but they are not owners or publishers and have no responsibility for its content or policies.

* Basil Gordon, Acting Managing Editor until February 1, 1966. 


\section{Pacific Journal of Mathematics}

\section{Vol. 15, No. $4 \quad$ December, 1965}

Robert James Blattner, Group extension representations and the structure space ........... 1101

Glen Eugene Bredon, On the continuous image of a singular chain complex .............. 1115

David Hilding Carlson, On real eigenvalues of complex matrices .................... 1119

Hsin Chu, Fixed points in a transformation group ............................. 1131

Howard Benton Curtis, Jr., The uniformizing function for certain simply connected Riemann

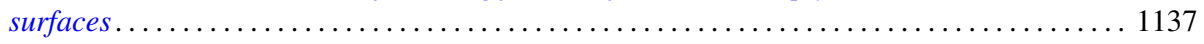

George Wesley Day, Free complete extensions of Boolean algebras................... 1145

Edward George Effros, The Borel space of von Neumann algebras on a separable Hilbert

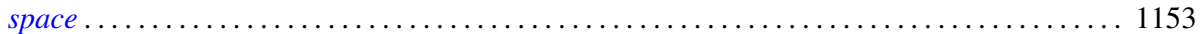

Michel Mendès France, $A$ set of nonnormal numbers ......................... 1165

Jack L. Goldberg, Polynomials orthogonal over a denumerable set ................ 1171

Frederick Paul Greenleaf, Norm decreasing homomorphisms of group algebras . . . . . . . . 1187

Fletcher Gross, The 2-length of a finite solvable group ........................ 1221

Kenneth Myron Hoffman and Arlan Bruce Ramsay, Algebras of bounded sequences ........ 1239

James Patrick Jans, Some aspects of torsion . . . . . . . . . . . . . . . . . . . . . . . 1249

Laura Ketchum Kodama, Boundary measures of analytic differentials and uniform

approximation on a Riemann surface ............................... 1261

Alan G. Konheim and Benjamin Weiss, Functions which operate on characteristic

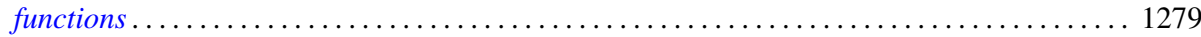

Ronald John Larsen, Almost invariant measures ............................ 1295

You-Feng Lin, Generalized character semigroups: The Schwarz decomposition ............ 1307

Justin Thomas Lloyd, Representations of lattice-ordered groups having a basis . . . . . . . . 1313

Thomas Graham McLaughlin, On relative coimmunity ....................... 1319

Mitsuru Nakai, $\Phi$-bounded harmonic functions and classification of Riemann surfaces ....... 1329

L. G. Novoa, On n-ordered sets and order completeness ..................... 1337

Fredos Papangelou, Some considerations on convergence in abelian lattice-groups . . . . . . . 1347

Frank Albert Raymond, Some remarks on the coefficients used in the theory of homology

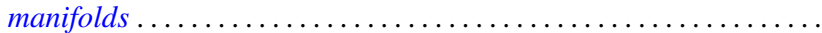

John R. Ringrose, On sub-algebras of a $C^{*}$-algebra .

Jack Max Robertson, Some topological properties of certain spaces of differentiable

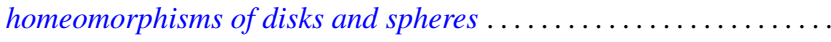

Zalman Rubinstein, Some results in the location of zeros of polynomials

Arthur Argyle Sagle, On simple algebras obtained from homogeneous general Lie triple systems. . . .

Hans Samelson, On small maps of manifolds ............................... 1401

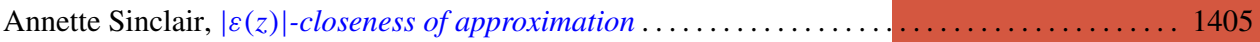

Edsel Ford Stiel, Isometric immersions of manifolds of nonnegative constant sectional curvature

Earl J. Taft, Invariant splitting in Jordan and alternative algebras ................. 1421

L. E. Ward, On a conjecture of R. J. Koch . . . . . . . . . . . . . . . . . . . . . . . . . . . 1429

Neil Marchand Wigley, Development of the mapping function at a corner . . . . . . . . . . 1435

Horace C. Wiser, Embedding a circle of trees in the plane ....................... 1463

Adil Mohamed Yaqub, Ring-logics and residue class rings . . . . . . . . . . . . . . . . 1465

John W. Lamperti and Patrick Colonel Suppes, Correction to: Chains of infinite order and their application to learning theory ........................................ 1471

Charles Vernon Coffman, Correction to: Non-linear differential equations on cones in Banach

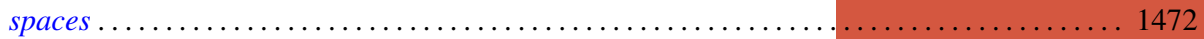

P. H. Doyle, III, Correction to: A sufficient condition that an $\operatorname{arc}$ in $S^{n}$ be cellular. . . . . . . . 1474

P. P. Saworotnow, Correction to: On continuity of multiplication in a complemented algebra 